Goldschmidt 2021 Abstract

https://doi.org/10.7185/gold2021.7521

\section{Impact of assimilating spaceborne lidar dust extinction in Northern Africa and Middle East}

JERÓNIMO ESCRIBANO ${ }^{1}$, ENZA DI TOMASO ${ }^{1}$, ORIOL JORBA $^{1}$, MARTINA KLOSE ${ }^{1,2}$, MARÍA GONÇALVES AGEITOS $^{1,3}$, FRANCESCA MACCHIA ${ }^{1}$, VASSILIS AMIRIDIS $^{4}$, HOLGER BAARS 5 , ELENI MARINOU ${ }^{4}$, EMMANOUIL PROESTAKIS ${ }^{4}$, CLAUDIA URBANNECK ${ }^{5}$, DIETRICH ALTHAUSEN ${ }^{5}$, JOHANNES BÜHL ${ }^{5}$, RODANTHI-ELISAVET MAMOURI ${ }^{6,7}$ AND CARLOS PEREZ GARCIA-PANDO ${ }^{1,8}$

${ }^{1}$ Barcelona Supercomputing Center (BSC)

${ }^{2}$ Karlsruhe Institute of Technology

${ }^{3}$ Polytechnical University of Catalonia (UPC)

${ }^{4}$ National Observatory of Athens

${ }^{5}$ Leibniz Institute for Tropospheric Research

${ }^{6}$ Cyprus University of Technology

${ }^{7}$ ERATOSTHENES Center of Excellence

${ }^{8}$ ICREA, Catalan Institution for Research and Advanced Studies

Presenting Author: jeronimo.escribano@bsc.es

Atmospheric mineral dust has a rich three-dimensional spatial and temporal structure that is poorly constrained in forecasts and analyses when only column-integrated aerosol optical depth is assimilated. This is the case for most of the current operational global aerosol products. Vertical aerosol information obtained from satellite-borne instruments can be assimilated in aerosol models, but questions about their consistency with the aerosol optical depth assimilation or the extent of their impact on the aerosol analyses remains --despite recent efforts-- an active subject of research. To contribute to these topics, we perform data assimilation with dedicated dust observations for a period of two months over Northern Africa, the Middle East and the Mediterranean sea. By means of a series of numerical experiments we study the added value of assimilating vertical dust information, with and without the joint assimilation of dust optical depth retrievals. Along with performing for first time the assimilation of the CALIOP-based LIVAS pure-dust extinction coefficient product in an aerosol model, we discuss the consistency of the assimilation of both sources of information and the role of the limited spatial coverage of the satellitederived pure-dust extinction coefficient within our assimilation scheme. In our experiments and for a similar spatial coverage, the assimilation of pure-dust profiles shows better error scores than the assimilation of dust optical depth, when they are compared with independent measurements. Best results are obtained when the full coverage of satellite-borne retrievals of dust optical depth is assimilated jointly with the dust extinction coefficients. By comparing with independent observations, we show that the vertical profiles of dust analyses are better represented in cases where dust extinction coefficient is assimilated, with and without the addition of dust optical depth observations; while the measured dust optical depth is better represented in experiments where dust optical depth is assimilated. We conclude that the assimilation of dust extinction coefficient profiles adds value to the analysis. New space-borne lidar missions could add value on desert dust forecasts if a depolarization capability is included, in order to discriminate dust and retrieve pure-dust extinction profiles that can be used for assimilation purposes. 